\title{
An unusual cause of recurrent hemoptysis: tracheopatia osteoplastica
}

\author{
Sandra Savelli • Maria Luisa Grata • \\ Alfonsina Ricci · Susanna Gamberini · \\ Roberto Manfredini
}

Received: 2 November 2009/Accepted: 1 February 2010/Published online: 23 February 2010

(c) SIMI 2010

An otherwise healthy 55-year-old man was referred to the hospital by his general practitioner due to recurrent episodes of hemoptysis. His medical history was silent, although his working activity (textile branch) exposed him to organic dusts. Shortly after an acute bronchitis treated with aspirin, the patient had a first episode of hemoptysis. Even after aspirin withdrawal, thoracic sense of oppression and cough persisted for several days, and a second hemoptysis event occurred. Cardiothoracic physical examination was unremarkable, and his general practitioner performed electrocardiogram, chest X-ray, and blood chemistry panel (all these examinations were normal), and asked for cardiologic and otorhinolaryngoiatric consultations, with no findings of pathological remarks.

Hemoptysis is a relatively common symptom of cardiopulmonary disease. Although bronchitis is a very common cause, a careful evaluation may be necessary to exclude more serious pathologic conditions, such as bronchogenic carcinoma and tuberculosis [1]. Moreover, the decision on whether to perform further investigations has not been influenced by the amount of bleeding. In fact, there is no correlation between quantity of blood and seriousness of the underlying thoracic disease [1].

In this case, given the patient's age, his working history, the recurrence of hemoptysis episodes, and prior negative

S. Savelli · M. L. Grata · A. Ricci · S. Gamberini

R. Manfredini $(\bowtie)$

U.O. di Medicina Interna, Ospedale del Delta,

Azienda U.S.L. di Ferrara, Ferrara, Italy

e-mail: r.manfredini@ausl.fe.it

R. Manfredini

Sezione di Clinica Medica, Dipartimento di Medicina Clinica e Sperimentale, Università di Ferrara, via Savonarola 9,

44100 Ferrara, Italy results, we decided not to dismiss hemoptysis as simply due to bronchitis, and proceed with a more advanced workup including CT scan and bronchoscopy. Thoracic high-resolution computerized tomography (HRCT) showed limited "polished glass" areas, localized to the posteriorbasal segments of inferior lobes. Bronchoscopy revealed the presence of multiple calcifications in the tracheobronchial cartilages, with normal mucosal surface, extending also to the larger bronchial ramifications and the carina (Fig. 1, upper and lower panels). Cultural examination and cytology of bronchoalveolar lavage were negative. The bronchoscopic picture was self-explaining, since the presence of sessile bony or cartilagineous nodules of the tracheal wall is the hallmark of tracheopatia osteoplastica.

Tracheopatia osteoplastica (or tracheopatia osteochondroplastica) (TO) is an uncommon disease of the upper airways, characterized by the presence of multiple cartilaginous or bony projections into the tracheobronchial lumen [2]. It usually affects people aged $>50$ years, but incidence and prevalence in the general populations are unknown, since the great majority of cases are diagnosed only post-mortem [3]. The cause is unknown, and more often this disease is asymptomatic. However, the most frequent symptoms at presentation are chronic cough (54\% of cases), sputum production (34\%), and hemoptysis (20\%) [2]. Computerized tomography may sometimes reveal tracheal soft tissue masses or beaded calcification in the tracheobronchial cartilages, although calcifications, luminal stenosis, wall thickening, and nodules need for differential diagnosis with other uncommon diseases involving the central airways, i.e., Wegener's granulomatosis, relapsing polychondritis, amyloidosis, sarcoidosis, and tuberculosis. Thus, bronchoscopy remains the main diagnostic tool: nodules are most frequently distributed along the proximal tracheobronchial tree, especially in the upper and middle 

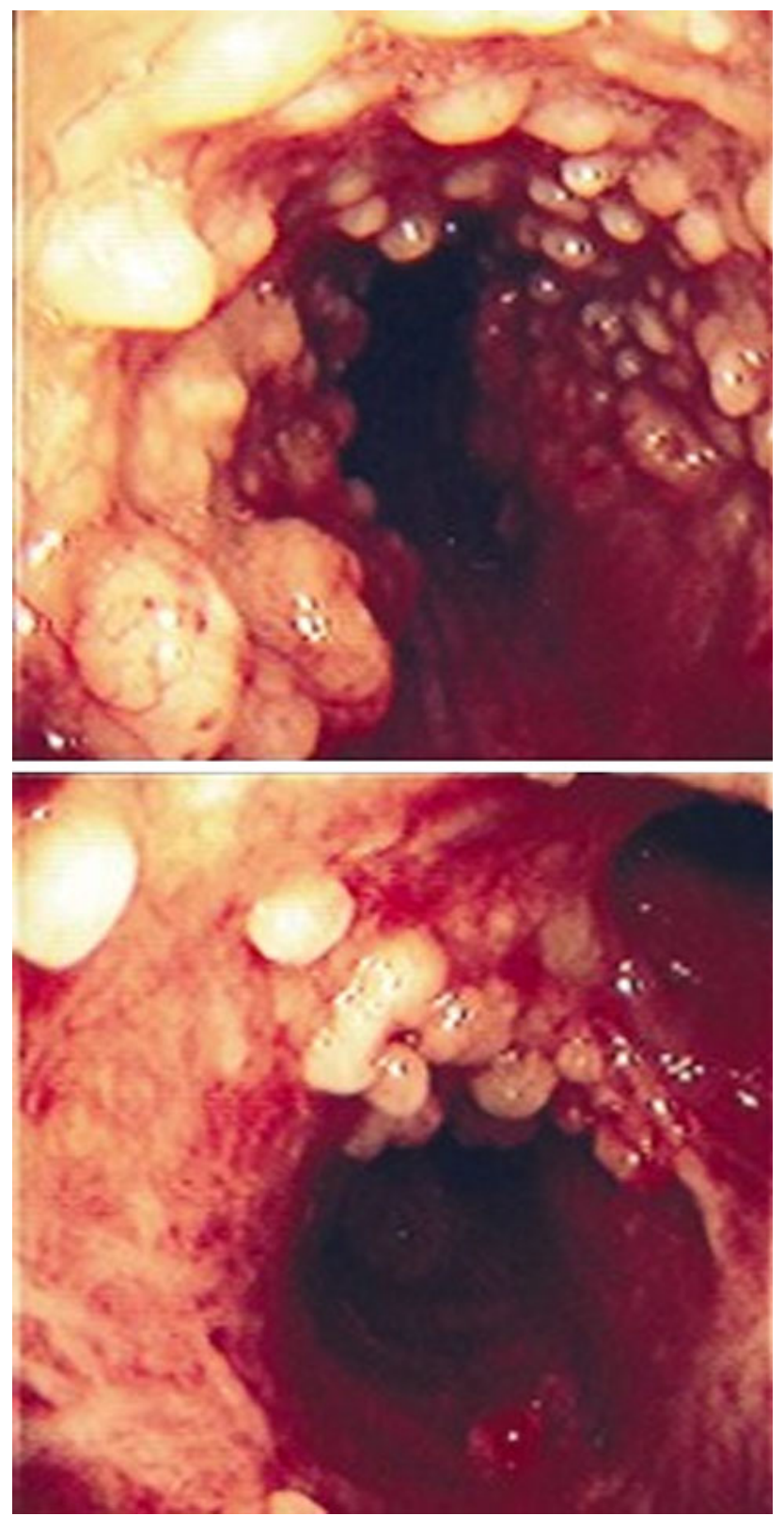

Fig. 1 Bronchoscopic images: multiple calcifications in the tracheobronchial cartilages, with normal mucosal surface, extending also to the larger bronchial ramifications and the carina trachea, affected in 76 and $78 \%$ of cases, respectively; the lower trachea is involved in $66 \%$, and the carina in $34 \%$ [2]. The main histopathologic findings include the presence of bone in the bronchial submucosa (56\% of cases) and squamous metaplasia of the tracheal epithelium (48\%) [2]. However, the characteristic histopathologic features of bony and cartilaginous nodules are most often obtained when analyzed at autopsy. In fact, endoscopists may have difficulty in performing a biopsy, since the nodules have a very hard consistency [2]. TO is not a static disease, since an endoscopic follow-up of $7 \pm 10$ years showed progression of lesions in $45 \%$ of patients (minimal in $28 \%$, significant in 17\%) [2]. The course is benign, and no specific treatment is currently available. However, since a possible complication may be purulent infection of the tracheobronchial tree, early introduction of antibiotic therapy would so be recommended in these cases. Although extremely unfrequent, cases of life-threatening airway obstruction and acute respiratory failure have been reported $[4,5]$.

\section{Conflict of interest None.}

\section{References}

1. Wolfe JD, Simmons DH (1977) Hemoptysis: diagnosis and management. West J Med 127:383-390

2. Leske V, Lazor R, Coetmeur D, Crestani B, Chattè G, Cordier JF (2001) Tracheobronchopatia osteochondroplastica: a study of 41 patients. Medicine (Baltimore) 80:378-390

3. Meyer CN, Dossing M, Broholm H (1997) Tracheobronchopatia osteochondroplastica. Respir Med 91:499-502

4. Molloy AR, McMahon JN (1988) Rapid progression of tracheal stenosis associated with tracheopatia osteochondroplastica. Intensive Care Med 15:60-62

5. Grillo HC, Wright CD (2005) Airway obstruction owing to tracheopatia osteoplastica: treatment by linear tracheoplasty. Ann Thorac Surg 79:1676-1681 